\title{
Educación del carácter: Participación social y cívica en el aula
}

Character education: social and civic participation in the classroom

\section{Volumen 18, Número 2}

Mayo-Agosto

pp. 1-24

Este número se publica el 1 de mayo de 2018

DOI: https://doi.org/10.15517/aie.v18i2.33159

\section{Eva Ortiz Cermeño}

\author{
Revista indizada en $\underline{\text { REDALYC, SCIELO }}$
}

Revista distribuida en las bases de datos:

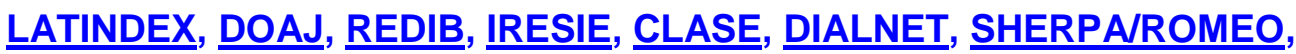
QUALIS-CAPES, MIAR

Revista registrada en los directorios:

ULRICH'S, $\underline{\text { REDIE}}, \underline{\text { RINACE}}, \underline{\text { OEI }}$ MAESTROTECA, PREAL, $\underline{\text { CLACSO }}$ 


\title{
Educación del carácter: Participación social y cívica en el aula
}

\author{
Character education: social and civic participation in the classroom
}

\section{Eva Ortiz Cermeño ${ }^{1}$}

Resumen: Este ensayo analiza la participación social y cívica del Centre for Narratives and Transformative Learning del Reino Unido (2005) y de la Comisión de Educación de los Estados Unidos (2013). En medio de un mundo, en continuo progreso y cambio, es preciso que, a través de la educación, se busquen rutas de pensamiento y acción que lleven hacia un concepto renovado y comprometido de ciudadanía, vinculado a otras definiciones (educación del carácter, ética, educación de la virtud) y basado en el respeto de los valores éticos universales. El objetivo del trabajo es ofrecer una revisión bibliográfica y descriptiva de los diversos autores que abogan por una educación ciudadana que contribuya a redefinir el espacio público, con lo cual se crea conciencia, intereses y pensamiento crítico en la sociedad. Se concluye que las escuelas tienen la responsabilidad de preparar al estudiantado para que desempeñe una ciudadanía participativa y adquiera valores para la convivencia democrática.

Palabras clave: participación estudiantil, sistema de valores, educación ciudadana, democracia.

Abstract: This essay analyzes the social and civic participation of the Center for Narrative and Transformative Learning of the United Kingdom (2005), and of the United States Commission of Education (2013), in the midst of a world in continuous progress and change where it is necessary that, through education you can find routes of thought and action that lead to a renewed and committed concept of citizenship. Linked to other definitions (character education, ethics, virtue education) based on respect for universal ethical values. The aim of the work is to offer a descriptive, bibliographic review of various authors who advocate that public education should contribute to redefining public space, creating awareness, interests, critical thinking in society. It is concluded that schools have the responsibility of preparing the student to perform a participatory citizenship and acquire values for democratic coexistence.

Key words: student participation, value system, citizen education, democracy.

\footnotetext{
1 Profesora asociada de Didáctica de las Ciencias Sociales de la Universidad de Murcia, España y Orientadora Pedagógica. Doctora en Pedagogía.
}

Dirección electrónica: evaortiz@um.es

Ensayo recibido: 2 de noviembre, 2017

Enviado a corrección: 20 de marzo, 2018

Aprobado: 23 de abril, 2018 


\section{Introducción}

El ser humano está teniendo una experiencia creciente a través de la historia. De acuerdo con Delval (2012) se debe fomentar en el estudiantado su capacidad de participación en la vida social en un sistema democrático, en el que haya igualdad de derechos y deberes para todas las personas, independientemente de su posición social o sus creencias. Por ejemplo, el estudio internacional sobre educación cívica y ciudadanía 2009 (ICCS) tiene como objetivo dar a conocer cuál es el desempeño del alumnado, basado en una prueba de comprensión y conocimiento conceptual relacionado con estos términos. Los datos del ICCS demuestran que:

Si bien muchos jóvenes se mantienen informados, valoran la intervención de los estudiantes, participan en actividades cívicas dentro del centro y tienen la intención de participar en elecciones y protestas legales, sólo unos pocos jóvenes tienden a involucrarse en organizaciones o grupos en su comunidad local, entienden bien la política o esperan participar activamente en la política a través de formas convencionales (Schulz, 2012, p. 47).

La sociedad democrática debe abogar por la cooperación e intervención de los educandos en asuntos públicos que contribuyan a su formación como ciudadanos de plenos derechos y deberes, (por ejemplo, participando en la elección de representante de curso, o de gobierno, en asociaciones o sindicatos estudiantiles, etc.)., rigiéndose por un marco normativo con medios que fluyan hacia la diversidad, la creación de organismos electorales e imparciales y hacia una ciudadanía informada y educada en asuntos decisivos para la responsabilidad política y ciudadana.

Como señala Gelpi (1992, p. 23), los valores determinan "la política, los contenidos y los métodos educativos de todos los sistemas escolares y la educación no formal". La preocupación que crece en la sociedad por ciertos fenómenos como el delito, la droga, la violencia, la intolerancia, etc., exigen de la escuela una acción que prepare a los educandos para enfrentarse a estos fenómenos sociales. Hay que fomentar la participación en el aula de los docentes-discentes sobre estos temas mediante cuatro competencias necesarias y primordiales: aprender a conocer, aprender a hacer, aprender a vivir juntos y aprender a ser, para potenciar la conciencia global en el mundo en el que nos encontramos inmersos.

La educación para la ciudadanía debería ayudar a redefinir el espacio público y promover la conciencia global y la cooperación. Hay diferentes significados de ciudadanía en 
una sociedad democrática y multicultural según Kymlicka (1995), Castles y Davidson (2000), Gutmann (1999), Rosaldo (1999) y Ong (1999), tales como: la educación de la virtud, educación ética, educación del carácter, educación cívica, etc.

Ten Dam y Volman (2007) explican que para trabajar la educación ciudadana y la educación del carácter se debe poner énfasis en la participación social y en la sociedad democrática y multicultural en las escuelas. Para sensibilizar al alumnado de los problemas reales que acontecen en el mundo exterior (contaminación, vandalismo, rebelión, consumo de sustancias, etc.), los docentes tenemos que ofrecer a los escolares una visión de conjunto de las materias impartidas, no solo a escala local sino global. Como también comentan (Boyd y Arnold, 2000, Naval, Print y Veldhuis, 2002, Print y Coleman, 2003 y Rychen y Salganik, 2003). Los ejemplos de virtudes que se transmiten en la educación del carácter pueden ser entre otros "ser honestos, esforzarse, obedecer a la autoridad legítima, amable, patriótica y responsable" (Jones, 2009, p. 39).

De acuerdo con Ehrlich (2000, citado en Browman, 2011, p. 30), el compromiso cívico debe ser trabajado mediante "la combinación de conocimiento, habilidades, valores y motivación. Significa promover la calidad de vida en una comunidad, a través de procesos políticos y no políticos". Es decir, tan importante es tener y desarrollar aptitudes (destrezas y competencias para ser buenos ciudadanos y ciudadanas), como actitudes y valores que los docentes tenemos que ejercer en la escuela, (respeto, tolerancia, empatía, responsabilidad, disciplina, libertad, igualdad, saber estar, etc.), para crear climas de convivencia pacíficos y democráticos en la colectividad a la que pertenecemos.

Hirmas y Eroles (2008) llevaron a cabo un estudio en México, Costa Rica y Chile sobre el entorno participativo en la convivencia escolar mediante valores y actitudes de cooperación y tolerancia en temas de inclusión, democracia y participación basados en la responsabilidad, como modos de proceder en la escuela y en la sociedad. Las experiencias aportadas muestran una mejora en las prácticas escolares de la comunidad educativa contribuyendo a la autonomía personal y al pensamiento crítico del alumnado. Acorde con Casares (2000), los valores no pueden separarse de la educación. La educación en valores es uno de los principales desafíos educativos del presente. Es importante coordinar y tratar de manera sistematizada los valores desarrollados a través del currículo para que no queden fuera de contexto, ya que se trata de un marco de referencia personal que guía a las personas en sus acciones, opiniones y decisiones (Paredes y Ribera, 2006) . 
Los docentes debemos educar con niveles de calidad, en asignaturas específicas de educación cívica, de historia y de gobierno, así como en los ejes transversales del currículum, supervisar el clima escolar, pedagógico y la organización escolar. En el estudiantado igualmente hay que promover el desarrollo de competencias (conocimientos, habilidades y actitudes), para participar cívica y políticamente.

Cortina (1997) se refiere a Scheler, Hartmann, Reiner y Hildebrand al comentar que es un mérito de la llamada ética de los valores haber intentado presentar clasificaciones de los mismos. Lo específico de estos valores es que dependen de la libertad humana y está en nuestra mano realizarlos.

La educación en valores también se espera que se exprese en el plan de estudios informal, por ejemplo en la gestión del aula, las interacciones entre maestros y estudiantes, la fijación de reglas, la participación de los estudiantes, la gestión de conflictos y contrarrestar el acoso y la discriminación (Thorberg y Oguz, p. 3).

Porque tan importante es saber transmitir y enseñar conocimientos de las diferentes disciplinas y materias como, normas de comportamiento, hábitos y actitudes para poder llevar a cabo de forma resolutiva una buena convivencia escolar dentro y fuera del aula, atendiendo a la diversidad y multiculturalidad fomentando la inclusión del educando.

De igual modo, la educación ciudadana en las sociedades latinoamericanas en general apuesta por una educación para el cambio en relación con los dilemas que nos encontramos cada día, partiendo de los problemas cotidianos y específicos de la realidad y del contexto, para que adquiera significado y sentido en la vida cotidiana del estudiantado (Torres, Álvarez y Del Roble, 2013).

\section{Desarrollo del tema}

\subsection{Civismo: espacio para la coexistencia común con los ciudadanos y ciudadanas}

La palabra civismo tiene la misma etimología que la palabra civilización, provienen del latín cives que significa ciudadano. Así, saber ser un buen ciudadano en una democracia es ser activo, reflexivo y crítico. El civismo intenta hacer el trato humano lo más agradable posible, a través de un comportamiento que respeta unas normas y unas costumbres sociales. Como argumentan Paredes y Ribera (2006), preocuparse por la tolerancia y la convivencia, reclamar respeto, intentar no herir susceptibilidades y limar la hostilidad en las 
relaciones entre las personas no debe dejar de lado, el preocuparse por conseguir una actitud más cívica en la sociedad actual.

Gutmann (2001) explica que una de las tareas fundamentales de la escolarización consiste en preparar a los miembros de cada nueva generación, en las responsabilidades que un día tendrán como ciudadanos/as. Tal y como enfatiza esta autora, la educación para la ciudadanía incluye, pero también supera, el marco de las clases de educación cívica dado que dicha educación, no estriba meramente en el aprendizaje de hechos básicos relacionados con las instituciones democráticas, ni en el de los procedimientos que permiten la vida política democrática de un país. Con este tipo de educación pretende estimularse la adquisición de disposiciones, excelencias cívicas (virtudes) y lealtades que se inculcan a lo largo de todo el proceso educativo, vertebrándose de tal modo los objetivos y los principios que ordenan el currículo.

Una escuela centrada en la formación política de sus actores ha de distinguir al menos dos aspectos de la educación: la formación institucional y el desarrollo de las virtudes cívicas. La educación cívica consiste, primordialmente, en el aprendizaje de la estructura, el funcionamiento de las instituciones, los procedimientos de la vida política y el cumplimiento de pactos sociales, a través del respeto activo como elemento fundamental para el desarrollo de los espacios y ambientes escolares. No se trata simplemente de tener buenos modales, o de "ser bien educado", sino que implica un compromiso con la no discriminación y un auténtico respeto por las diferencias. De acuerdo con Martínez Martín (2011, p.1):

La educación de los sujetos como individuos éticamente autónomos, políticamente conscientes, socialmente comprometidos resulta una necesidad crecientemente reconocida en el marco de nuestras sociedades democráticas, ante la que la escuela tiene mucho por hacer si lo que se quiere es contrarrestar la racionalidad puramente instrumental imperante en nuestra época que privilegia valores efectivistas e individualistas.

En los espacios educativos, cuando los docentes-discentes estamos interactuando en el aula, a través de la participación ciudadana, como expresa Kymlicka (2001, p. 261), "la auténtica civilidad no significa sonreír a los demás sin que te importe cómo te maltratan [...]. Significa, más bien, tratar a los demás como iguales, con la condición de que aquellos lo hagan también contigo". Pero incluso si se es objeto de maltrato, esto no legitima el derecho a responder de la misma manera. En la formación de la civilidad, el debido respeto por el 
otro, el reconocimiento y aprecio de la diversidad permiten contrarrestar la idea de que en la escuela y en la sociedad, todos tienen que expresarse de manera homogénea, lo cual tiene poco o nada que ver con la idea de la igualdad.

Tanto la ciudadanía, como los valores de la educación en general y el carácter de la educación en particular han producido cierta preocupación en el siglo XX. Lickona (1991) se refiere a distintos fenómenos que producen un brote de pánico en América como: el crimen, el divorcio, el vandalismo, el alcoholismo, el uso de drogas, el aborto, la violencia, la falta de honestidad y honradez, así como el fraude en los negocios y los impuestos.

En el desarrollo de las virtudes cívicas, además de las virtudes estándar de la veracidad y la honestidad, la honradez y el cuidado hacia los otros, socialmente aprobadas para ser inculcadas se han incluido otras como: el sentido del orden, la ciudadanía, la castidad y el patriotismo. Wringe (2006) hace mención a algunos escritores como Schubert, Lickona y Purpel que tienden a usar el término "educación para el carácter", de forma amplia para referirse al civismo, o a la educación en valores en general.

Lockwood (2003), por el contrario busca unos objetivos conductuales más explícitos, una definición orientada hacia la formación del comportamiento, por medio de valores valores democráticos de: respeto, tolerancia, responsabilidad, trabajo en equipo, igualdad, equidad, solidaridad entre otros, que se potencian en los espacios de aula con ejercitación de las habilidades para su desarrollo y fortalecimiento mediante estrategias de mediación del aprender a aprender para el logro de procesos de meta cognición.

Lickona (1991) argumenta que son sumamente influyentes en la educación para el carácter, las acciones y el conocimiento de la formación cívica, el desarrollo de la moralidad (la conciencia, la empatía, el amor por lo bueno y la humildad). El carácter, explica el autor, consiste en valores operativos, es decir, valores en acción.

Como recoge la Unesco (1993), la educación para los derechos humanos y para la democracia es en sí un derecho fundamental y una condición esencial para la justicia social, la paz y el desarrollo. El ejercicio de ese derecho contribuirá a preservar la democracia y a asegurar su desarrollo en el más amplio sentido. Schemelkes (1997) argumenta que existen diversos planteamientos acerca de las razones de fondo, por las que debe plantearse la necesidad de la formación en valores, o ética.

Schemelkes (1997) alude a Savolainen, para comentar que hay una preocupación generalizada por los nuevos problemas éticos que surgen debido al desarrollo de la ciencia y la tecnología (la bioética y la genética, por ejemplo). Los educandos deberían ser informados 
de los nuevos descubrimientos, para que tengan la oportunidad de considerar las consecuencias de estos desarrollos, desde un punto de vista de la formación cívica. Ambos aspectos tienen que ver con la calidad de vida actual, e inclusive, con el futuro de la humanidad. Conforme las sociedades se van haciendo cada vez más internacionales y multiculturales es necesario desarrollar nuevas formas, para que esta diversidad se convierta en una fuente de riqueza, en lugar de una fuente de tensión y conflicto. El respeto universal a los demás, especialmente allí donde existe diversidad cultural debe incorporarse a la actividad educativa de las personas.

La combinación de la restauración de la misión cívica de los colegios con la educación global puede conducir a un aprendizaje transformador global y local. Si las iniciativas mundiales y locales se pueden infundir a principios de la carrera universitaria de los estudiantes, ese aprendizaje puede convertirse en un elemento central de la experiencia intelectual y personal formativa de todos los estudiantes, en lugar de la experiencia marginal de unos pocos. (Boyle, 2014, p. 75)

Para Pérez y Mendoza (citado en Schemelkes, 1997), los espacios de socialización de los individuos (familiares, escuela, entorno) se reconocen importantes para la formación en valores. Sin embargo, es la escuela la que permite trabajar de una manera intencionada y sistemática las temáticas relacionadas con ello. Tirado (citado en Schemelkes, 1997) considera que si la escuela no forma en valores deja de cumplir la función socializadora, que es inherente a ella. En una sociedad democrática, ¿quién forma para la democracia? La función socializadora de la escuela implica reconocimiento y valoración del pluralismo. Así como formar, para participar y ejercer el juicio crítico.

Las escuelas deben ayudar a los estudiantes a entender cómo las identificaciones culturales, nacionales, regionales y globales están interrelacionadas, complejas y en evolución. Estas identificaciones son interactivas de una manera dinámica. Cada uno debe ser reconocido, valorado, públicamente afirmado, y cuidadosamente examinado en las escuelas. Los estudiantes deben ser alentados a examinar críticamente sus identificaciones y compromisos y comprender las complejas formas en que están interrelacionados y construidos. (Banks, 2004b, citado en Banks, 2008, p.134)

Hay que llevar a cabo en las aulas una educación integradora, inclusiva para concienciar al estudiantado sobre la diversidad, independientemente de sus condiciones 
laborales, personales, culturales o sociales, como elemento enriquecedor del proceso de enseñanza aprendizaje y, en consecuencia, favorecedor para el desarrollo humano.

Zorrilla (citado en Schemelkes, 1997) parte de la convicción de que la educación cívica es vital porque un estado de autonomía es mejor que uno de heteronomía implicando un papel más activo del sujeto sobre su desarrollo, una mayor profundidad y un significativo sentido de la responsabilidad. La educación para la ciudadanía contribuye a que los ciudadanos y ciudadanas debemos no solo ser sujetos de reconocimiento de las identidades personales, sino también que seamos capaces de disfrutar de la diversidad del otro, para desarrollar una conexión humana entre las personas alrededor del mundo. Las identidades, los apegos y los compromisos globales constituyen el cosmopolitismo (Nussbaum, 2002).

La educación, como afirma Savolainen (citado en Schemelkes, 1997), no constituye solamente un derecho humano, sino que tiene que representar el medio por excelencia para la ejercitación de las capacidades ciudadanas en los espacios del aula, con el fin de mejorar la calidad de vida. Algunos de los objetivos de la educación para el desarrollo del juicio de la formación cívica y la educación para el carácter son, como sostiene Zvokey (1990), entre otros:

1. Tomar decisiones satisfactorias y acciones efectivas en situaciones cívicas.

2. Elegir los valores que van a formar sobre futuras decisiones y acciones participando socialmente.

3. Resolver conflictos, es decir, situaciones en las que intervienen principios de formación cívica excluyentes (paz vs. justicia, por ejemplo).

4. Llegar a principios de formación cívica que todos siguieran (principio de la universalidad).

5. Que los niños y niñas sean conscientes de las consecuencias de sus actos (responsabilidad).

6. Lograr consistencia entre lo que se valora y cómo se actúa.

7. Preparar para participar de forma activa y con una ética ciudadana en la sociedad democrática.

8. Ayudar a las personas a valorar el pluralismo.

En la escuela, las prácticas incluirán una amplia gama de actividades pedagógicas más allá de la instrucción didáctica, como el juego, la investigación, el debate, el diseño, el desempeño, los deportes, etc., Cada actividad pedagógica tiene desafíos y beneficios 
únicos. Las prácticas pueden presentar características tales como: mentalidad de crecimiento; etapas del desarrollo ético; cooperación (en deportes, música, robótica, etc.) y colaboración (equipo estructurado); experimentación segura, con esfuerzos que estimulan al estudiante; Procesos, no sólo de conocimiento sino sistematicamente metacognitivo (reflexión sobre procesos); extensión longitudinal/ plurianual (de proyectos, y planificación de carrera); participación de los mayores en dinámicas mixtas (sabiduría, sensibilidad, etc.); prácticas/capacitación laboral; incorporación de la tecnología. (Bialik, Bogan, Fadel y Horvathova, 2015, p. 3)

Según Aspin (2000), los valores son principios particulares, o normas de conducta, por los que una persona busca o decide vivir. No son ni privados, ni subjetivos, son objetivos y públicos. Se puede hablar de todos ellos, rechazarlos o aprobarlos. Los juicios de valor constituyen puentes entre nosotros en cuanto a los caminos en los que debemos actuar, 0 las cosas que debemos admirar.

\subsection{Argumentos teóricos y metodológicos. La participación en la escuela. Un ejercicio de voluntades y compromisos éticos}

Participar etimológicamente procede del latín participare, que en un sentido activo del verbo significa "tomar parte", y en un sentido causativo "hacer tomar parte". Este último vendría a completar la acción de dar, con la de recibir en la participación (Naval, 2003). Formar ciudadanos significa:

No sólo enseñar un conjunto de valores propios de una comunidad democrática, sino estructurar el centro y la vida en el aula con procesos (diálogo, debate, toma de decisiones), en los que la participación activa, en la resolución de los problemas de la vida en común, contribuyen a crear los correspondientes hábitos y virtudes cívicas. (Bolívar, 2007, p. 23)

En necesario en el aula crear espacios abiertos para que el estudiantado manifieste sus puntos de vista, argumente, reflexione sobre los temas expuestos a través de una participación democrática e interactuando con los compañeros y compañeras, para construir el conocimiento aprendido mediante el razonamiento, la cognición y el aprendizaje significativo. 
Organizar la vida escolar en régimen participativo y especialmente la participación activa del alumnado en la vida escolar es importante para la formación cívica (Lorenzo, 2000). Camps y Giner (1998) argumentan que la democracia precisa ciudadanos/as, esto es, personas que quieran colaborar en la construcción y mejora de la vida colectiva. Sin personas responsables no hay democracia auténtica. Hay que desarrollar y tener en cuenta diferentes técnicas para promover la participación con los estudiantes. Thomas (2002, p. 440) destaca entre otras:

La ampliación de la participación es una actividad de toda la institución y no se limita a proyectos específicos (proyectos a corto plazo), o la responsabilidad de unos cuantos que "cuidan". Liderando el ejemplo: el personal superior debe demostrar su compromiso de ampliar la participación y estructurar sus propias relaciones con los profesionales y con los estudiantes de una manera que demuestre el respeto por la diferencia. Desarrollo del personal para promover actitudes inclusivas y procedimientos de enseñanza, aprendizaje y evaluación. Medidas de validación y aseguramiento de calidad que enfatizan una pedagogía colaborativa y un currículo inclusivo. Una revisión de las opciones de alojamiento disponibles, y la medida en que los estudiantes son capaces de moverse.

Díaz (2000) afirma que la democracia como sistema político implica una doble participación. Por un lado, una participación libre en la toma de decisiones y por otro, una participación efectiva en los resultados, es decir, en derechos y libertades. Ambas exigencias se corresponden con la democracia, de forma cívica, autonomía ética individual y autorrealización personal, es decir, el ser humano como un ser de fines, y con la democracia como Estado de Derecho, imperio de la ley, procedimientos de expresión de todas las personas en la formación de la voluntad general y protección-realización de los derechos fundamentales.

En Costa Rica el concepto de educación cívica ha evolucionado, pasando por espacios de carácter político y patriotismo, para entrar en una discusión necesaria en el presente. Se lleva a cabo desde un enfoque curricular academicista, con objetivos y ejes temáticos estrechamente relacionados con la realidad mediata e inmediata del estudiantado. En este sentido, la unión de los términos, educación y cívica, es conveniente valorarlos como parte de una estructura, donde convergen diversidad de elementos que no se deben obviar en 
teoría. Hacen referencia a procesos, etapas, sistemas, intenciones, actitudes y necesidades entre las personas, los pueblos y las sociedades (Alfaro y Badilla, 2013).

La democracia está bien orientada cuando descansa sobre el concepto de participación cívica, en lo que se refiere a cuestiones de dominio social. La participación es la razón de ser de la democracia. Es esencial para que las democracias sean viables, sostenibles y saludables (Crick, 1998, 2002; Norris, 2002 y Putnam, 2000). Pero la participación es un concepto, como explica Naval (2009), no meramente procedimental.

Es a través de un sentido de responsabilidad personal y ética que los estudiantes, los ciudadanos/as del futuro, serán capaces de tomar decisiones que aborden los desafíos anteriores. Estos son los objetivos generales de la educación del carácter: construir una base para el aprendizaje permanente; apoyar las relaciones exitosas en el hogar, en la comunidad y en el lugar de trabajo; desarrollar los valores y virtudes personales para la participación sostenible en un mundo globalizado. (Bialik et al., 2015, p. 2)

La democracia costarricense es un régimen presidencialista estable con un estado cuyas decisiones están agrupadas en las entidades del gobierno central. La organización del Estado está tutelada por la Constitución Política de 1949 y las reformas posteriores. Dentro de los cánones de un Estado republicano organizado bajo un régimen presidencialista, común a la mayoría de los países latinoamericanos, el texto constitucional distingue tres poderes (ejecutivo, legislativo y judicial), a los que da soberanía e independencia, al tiempo que establece controles recíprocos.

Sin embargo, el régimen democrático costarricense se diferencia de sus análogas en la región por: la trascendente expansión en el reconocimiento de los derechos a la población, a partir de la base previa de carácter poliárquico; un reforzamiento de los dispositivos de protección y tutela de estos derechos; y por los cada vez numerosos y potentes controles políticos, legales y administrativos que recaen sobre el ejecutivo, tendencias características de las últimas décadas del siglo $X X$. En el presente, múltiples actores políticos e institucionales, incluso la ciudadanía en general, tienen (por lo menos alguna) capacidad efectiva de vetar la formulación o ejecución de políticas públicas (Vargas, Rosero y Seligson, 2006).

Lo importante no es simplemente participar por participar, como si fuera un simple juego, sino saber en qué se participa, por qué y para qué se implica alguien en actividades comunes que afectan a la vida del conjunto de la sociedad, y para las que nuestra 
colaboración personal es importante, es más, es insustituible. Marchesi (2000, p. 148) formó parte en la reforma educativa, con la Ley Orgánica General del Sistema Educativo en España (LOGSE, 1990), y dice a este respecto:

He de reconocer que la participación del alumnado es a veces muy complicada, lleva mucho tiempo y no consigue de forma inmediata los efectos deseados. Sin embargo es una de las estrategias más positivas para conseguir crear escuelas de ciudadanía, en las que el alumnado se sienta respetado y con la obligación de respetar a otros.

Los cauces de participación y de acción ciudadana, desde el punto de vista de la intervención pedagógica y la legitimación de medidas exigen que se genere una responsabilidad social y jurídica compartida, hacia la construcción de experiencias axiológicas, respecto de la convivencia pacífica y los valores derivados de los derechos humanos, en el marco constitucional que garantiza derechos y libertades (Touriñán, 2010).

El ejercicio de la ciudadanía requiere seres humanos autónomos en el cumplimiento de los deberes y los derechos para la vida en comunidad. Asimismo, la educación para la participación ciudadana implica capacidad de aportación, en la solución de problemas políticos y sociales con sentido de solidaridad y responsabilidad, y debe propiciar el desarrollar capacidades en el estudiantado para el ejercicio de la ciudadanía activa. Acorde con Gutiérrez y Arana (2012, p. 437):

Un reto de la escuela es la formación de la autonomía, en la que el juicio libre vincule el interés propio con el de los otros, con la distribución equitativa, la cooperación, la participación igualitaria, y con la determinación de objetivos comunes establecidos democráticamente.

En los documentos de la política educativa del Consejo de Europa prevalece la expresión Educación para la Ciudadanía Democrática. En ese contexto, el interés por la educación ciudadana fue abriéndose camino lentamente hasta el punto de lograr que en 1997, una declaración del Consejo de Europa de Jefes de Estado y de Gobierno, la estableciese como prioridad principal de su programa político y educativo. En América Latina (Argentina, Bolivia, Brasil, Chile, Colombia, Costa Rica, Ecuador, El Salvador, Guatemala, Honduras, México, Nicaragua, Panamá, Paraguay, Perú, República Dominicana, Uruguay, Venezuela) conversar sobre ciudadanía integral es considerar que los ciudadanos y 
ciudadanas deben acceder armoniosamente a sus derechos cívicos, sociales, económicos y culturales, y que ellos conforman un conjunto indivisible y articulado (Caputo, 2004).

Las relaciones a nivel regional entre la Unión Europea y las seis repúblicas centroamericanas se han suscrito históricamente en el Diálogo de San José, comenzado en 1984 en Costa Rica. Constituye el principal dispositivo de diálogo político entre las dos regiones. Fue establecido para asentar el proceso de paz y la democratización de la región. Tras su renovación en 1996 y en 2002, el Diálogo de San José se acrecentó al desarrollo económico y social. En 2006 tuvo lugar en Viena la cuarta Cumbre entre los jefes de Estados y de Gobiernos de los países latinoamericanos, del Caribe y de la Unión Europea, durante la cual se ratificó el compromiso de fortalecer la colaboración biregional. Asimismo se matizó sobre el multilateralismo, la integración regional y la cohesión social como prioridades del diálogo político y de la cooperación (Informe estratégico nacional de Costa Rica, 2007-2013).

En 2012, una nueva Recomendación del Consejo de Europa (2012) sugirió algunos parámetros adicionales. Entre ellas, se alienta a los Estados Miembros a que incluyan programas de ciudadanía democrática en todas las formas de educación y formación, ya sean formales o informales, incluso a nivel de educación superior, en particular en los cursos de formación de profesores. Una de las principales razones para ello es reforzar la capacidad de los estudiantes para participar en la sociedad. También se alienta a los Estados miembros a que desarrollen los criterios necesarios para evaluar estos programas, así como para iniciar y desarrollar investigaciones sobre prácticas innovadoras, metodología de enseñanza y estrategias de evaluación. (Arbués, 2014, p. 227)

En las fuentes legislativas nacionales según Gómez Rodríguez (2005) se pueden encontrar distintas acepciones de educación para la ciudadanía. Por ejemplo, participación cívica (Letonia, Rumania), aptitudes cívicas y conciencia social (Polonia), compromiso en la formación cívica (Rumania), derechos y deberes de la formación cívica (Alemania, Países Bajos, Reino Unido, España, Italia, etc.). Todos los países con énfasis variable asocian el concepto de ciudadanía a valores comunes como democracia, dignidad humana, respeto de los derechos humanos, tolerancia, igualdad, solidaridad, cooperación, cultura política, europeísmo, etc., sin olvidar el desarrollo espiritual, la formación cívica y cultural de la persona. 
En 1978, teóricos políticos, como por ejemplo Gunsteren mostraron un gran interés por el concepto de ciudadanía. Más tarde, Heather (1990) sostiene que este concepto llegó a ser la, buzz Word, entre los pensadores de los puntos del espectro político. El interés por la ciudadanía ha sido provocado por diversos acontecimientos políticos y tendencias en todo el mundo: el aumento de apatía del votante, y la dependencia de bienestar a largo plazo en Estados Unidos, el resurgimiento de movimientos nacionalistas en Europa Oriental, la presión creada por una población cada vez más multicultural y multirracial en Europa Occidental, el fracaso de la política ambiental que confía en la cooperación ciudadana voluntaria., etc.

La educación tradicional de la ciudadanía (típicamente autoidentificada como "educación cívica") ha empleado el modelo de conocimiento. En los cursos de educación cívica que se requieren en los programas de estudios de los centros de enseñanza media y especialmente de las escuelas secundarias, el enfoque se centra en el conocimiento fáctico sobre el gobierno, así como sobre el individuo como portador de derechos constitucionales. (Althof y Berkowitz, 2006, p. 502)

Acorde con lo anterior, Garnier (2013) en la República de Costa Rica, llevó a cabo unos programas de estudios sociales y educación cívica, en los que resalta que, hay valores que son esenciales en la formación ética y ciudadana, la justicia y la equidad; la autonomía, como antítesis del autoritarismo; la tolerancia y el respeto a la diversidad; la difusión de la libertad en su sentido amplio de capacidad, soy libre cuando tengo la libertad real de ejercer mis capacidades y mi potencial; la dignidad como derecho a una vida plena. La solidaridad y la simpatía, la identificación con el otro y la responsabilidad entre todas las personas y con el entorno.

\subsection{La educación para la ciudadanía y la formación del carácter en el Centre for Narratives and Transformative Learning del Reino Unido y la Comisión de Educación de los Estados Unidos}

Halstead y McLaughlin (1999) exponen que la educación para la ciudadanía no es simplemente una materia de conocimiento sobre instituciones políticas y principios constitucionales, sino también se refiere a cómo pensamos y nos comportamos hacia los otros, y en particular lo que nos diferencia respecto a la raza, clase o religión, inculcando 
hábitos, virtudes e identidades. La ciudadanía requiere cultivar el hábito de la cortesía y la capacidad para el razonamiento público en nuestra interacción con las personas.

La escuela americana es partícipe de un sistema de educación pública descentralizada que comprende aproximadamente 15200 distritos escolares, cada uno de los cuales determina su propia política y prácticas para dirigir la educación para la ciudadanía. No es sorprendente que existan amplias variaciones entre los distritos respecto a los planes de estudios, los salarios del profesor, los gastos por alumno/a, la calidad de la educación y las tarifas de graduación. Ichilov (1998) comenta que, el sistema escolar primario y secundario es principalmente responsable de enseñar la ciudadanía a los estadounidenses. Imbernón (2004, p. 80, citado en De Castro y Da Silva, 2014, p.1686) afirma que:

No es sólo un entrenamiento como una colección de técnicas y procedimientos, sino que tiene una carga ideológica, valores, actitudes y creencias. Es importante recordar que no se trata de un simple cambio [...] de enfoque para redefinir el contenido, las estrategias, los protagonistas y los propósitos de la formación. Por lo tanto, pretende desarrollar una cultura colaborativa y participativa, permitiendo: procesos de acciónreflexión-acción; cambio de realidad cultural; nuevos valores: proponer interdependencia, apertura profesional, comunicación, acto educativo de hacer acto público, colaboración, autonomía, autorregulación y crítica colaborativa; colaborar no como una estrategia de gestión, sino como una filosofía de trabajo; procesos de participación, propiedad y pertenencia; respeto y reconocimiento del poder y la capacidad de los docentes; búsqueda de poder y desarrollo por parte de los profesores; reajuste y expansión de la gestión escolar.

En general, los niños y niñas de la escuela primaria son expuestos a símbolos y rudimentos de la historia y del gobierno estadounidense. En muchos Estados, también se les enseña sobre su gobierno estatal, la historia y los símbolos (la bandera estatal, la canción, la mascota y la flor). Para socializar a los jóvenes en la democracia estadounidense se incluyen las elecciones oficiales de clase, que votan sobre el aula, o las decisiones de la escuela. La instrucción formal más intensiva en la educación ciudadana por lo general ocurre en la escuela secundaria (Junior High School).

Muchos Estados y localidades requieren que los estudiantes realicen un curso sobre educación cívica, los principios y problemas de la democracia, el gobierno, etc. Este curso básico es apoyado por cursos anteriores y subsecuentes, sobre todo en los estudios sociales 
y de historia. El contenido del curso trata principalmente sobre la historia estadounidense, la ciencia política, el gobierno, la economía, los problemas sociales, el derecho y la sociología. La educación para adultos es otra vía para promover la educación para la ciudadanía. Una gran variedad de grupos y organizaciones (sindicatos, asociaciones patrocinadas por el gobierno, los partidos políticos, etc.) ofrecen un entrenamiento formal, o los talleres que realizan los adultos desarrollando conocimiento, destrezas, aptitudes y comportamientos sobre ellos mismos y su rol de ciudadanía.

La Comisión de Educación de los Estados Unidos (2013) comenta que, preparar al estudiantado para que participen social y cívicamente en la nación de la que forman parten es uno de los fines fundacionales de la educación pública en este país. Las escuelas públicas permanecen como la mejor vía para garantizar que los ciudadanos y ciudadanas posean los conocimientos, habilidades y disposiciones necesarias, para la participación productiva en la vida cívica y política. Si bien el papel que desempeña la educación cívica en las escuelas públicas se ha reducido en los últimos 50 años, este ha seguido haciendo progresos en sus prácticas educativas.

Desde la visión de la Universidad Cívica en Inglaterra y EE.UU podemos ver claramente que la definición de "cívica" aquí no es simplemente el uso de las instalaciones de infraestructura urbana. En otras palabras, se enfatiza la relación entre la Universidad y la Ciudad y la conciencia cívica educativa para el aprendizaje permanente. La misión principal de la universidad es impartir conocimientos, explorar la investigación y humanizar a los ciudadanos. En la era de la globalización y la información, la universidad se convirtió en una comunidad académica intensamente vinculada con la ciudad [...]. Más específicamente, desde una perspectiva de política de educación superior, el compromiso con una ciudad puede proporcionar un signo externo y visible de la contribución de la universidad a la sociedad civil. El concepto de la Universidad Cívica derivado del Reino Unido en el siglo XIX da respuesta a los retos de la rápida industrialización y urbanización. (Wu y Oldfield, 2015, p.13)

La educación para la ciudadanía en las escuelas estadounidenses es difícil porque hay controversias inherentes en la democracia (la libertad contra la igualdad, el gobierno mayoritario contra los derechos de las minorías, etc.). Por ejemplo, mientras muchos administradores de la escuela, el profesorado y los padres creen que el objetivo principal es promover ciudadanos y ciudadanas bien formados, convencionales y atendidos, otros 
adultos demandan personas críticas orientadas a resolver problemas para desarrollar un cierto activismo. Otro ejemplo de Educación para la ciudadanía es el caso del Reino Unido de la Gran Bretaña, e Irlanda del Norte. Hay varios factores a destacar en este Estado sobre la educación para la ciudadanía.

Ichilov (1998) argumenta que el Reino Unido ha tenido, y todavía tiene, una monarquía y algunos argumentan que la ciudadanía es esencialmente un concepto republicano (Estados Unidos y Francia, como ejemplos principales). El futuro de la monarquía es un tema de debate abierto. No tiene ninguna constitución escrita, y ninguna declaración de derechos, ni ningún código oficial en el que la ciudadanía pueda ser relacionada en términos de derechos y responsabilidades. La reforma constitucional está ahora en la agenda de los partidos políticos.

El Reino Unido está compuesto por cuatro países: Inglaterra, Escocia, Gales e Irlanda de Norte. Esta "unión" podría ser una declaración de intención más que una declaración de hecho. Y la ciudadanía está asociada normalmente, con el concepto de nación. Desde 1945, con el establecimiento de antiguos pueblos coloniales ha llegado a ser una sociedad multiétnica y multicultural (como Estados Unidos, Canadá y Australia, sus antiguas colonias). Plantea preguntas sobre cómo compaginar códigos de formación cívica múltiples (cada uno con su propia integridad) dentro de la misma sociedad.

Los ingleses eran los vencedores evidentes de las guerras regionales. La lengua inglesa era el lenguaje de poder y de gobierno. Escoceses e irlandeses fueron sometidos a las costumbres inglesas, y esto plantea problemas para la ciudadanía como una identidad compartida. La sociedad inglesa tiene una conciencia de clase. El sistema de educación formal ha sido dual, de élite y de instituciones de masas. Los reformadores de la educación progresiva se han esforzado por democratizar el acceso al conocimiento (con buen éxito) y democratizar las instituciones (con menos éxito).

Como recoge, el Centre for Narratives and Transformative Learning (2005), las escuelas en el Reino Unido tienen la responsabilidad de preparar a la gente joven para desempeñar un papel eficaz en la sociedad, hacer buenos ciudadanos/as, mientras también reconocen el papel vital de los padres como educadores principales. Esto se manifiesta de dos formas. En primer lugar, desde el año 2000, las escuelas han sido requeridas para que la educación para la ciudadanía forme parte del Plan de Estudios Nacional en Inglaterra, para todo el alumnado en las etapas claves 1-4. En segundo lugar, los estudiantes aprenden 
sobre la ciudadanía por experiencias en la acción, a través de las cuales ellos son enseñados y sus vidas (en las escuelas) son organizadas.

Los estudios de formación cívica en este centro reflexionan principalmente en lo estructural, procesal y en los aspectos legales de las instituciones políticas evitando la discusión de polémicas y acentuando el acuerdo general, la armonía y el cumplimiento. Para que los educandos se integren, participen, cooperen en el aula y se formen en valores cívicos (respeto, tolerancia, empatía, alteridad, justicia, igualdad, etc.), los docentes abordan temas como la educación para la paz, la educación multicultural, la educación de los derechos humanos, la educación para el desarrollo, la educación ambiental y la educación global. La educación para la ciudadanía necesita proporcionar un sentido objetivo, de solidaridad y dirección en un mundo fragmentado que rápidamente cambia tal y como se indica en la tabla 1.

Tabla 1

Objetivos educacionales

- Prevenir la experimentación y abuso de drogas, alcohol y otras sustancias tóxicas.

- Desarrollar la autoestima.

- Analizar las capacidades de resolución de problemas y de toma de decisiones.

- Maximizar el uso del tiempo y talentos.

- Interpretar el respeto a los derechos de los otros. Identificar las obligaciones y derechos de un ciudadano.

- Trabajar de forma cooperativa.

- Aprender a ocuparse con autodisciplina para lograr objetivos concretos.

- Distinguir entre el bien y el mal.

- Asumir responsablemente las acciones propias.

- Resistir la presión negativa de los pares.

Fuente: Character Education Institute (2005)

Ciertamente las ciencias del aprendizaje hacen hincapié en la importancia de la organización comunal de las escuelas, la pertenencia social, la prensa académica y las teorías constructivistas cognitivas del aprendizaje y la instrucción, y éstas son las metas de la formación de los profesores. Si la educación del carácter es sólo una buena educación, los maestros y los líderes escolares que están capacitados en estos asuntos también están aprendiendo a dirigir los resultados del comportamiento de los estudiantes hacia fines deseables; $Y$ estos fines se considerarán marcas de buen carácter. (Lapsley y Woodbury, 2016, p.197) 


\section{Síntesis y reflexiones finales}

De acuerdo con Annette y Mayo (2010), la participación cívica y social se tiene que considerar una dimensión importante en la instrucción de los educandos. De igual modo, expresan que el desarrollo del aprendizaje de la formación cívica es relevante, y que los docentes en los procesos de enseñanza deben transmitir y enseñar valores y prácticas de ciudadanía democrática.

Hay que trabajar con los estudiantes no sólo el entorno próximo, sino aplicar el aprendizaje de forma global, traspasando todas las fronteras, nacional e internacionalmente para que se integren en la sociedad, y entiendan su país y el resto de comunidades participando activamente en la sociedad como ciudadanos de plenos derechos y deberes. Tener un conocimiento profundo, no sólo teórico sino práctico de las materias que se enseñan, así como la calidad en el aprendizaje es vital para que las nuevas generaciones que terminan sus estudios universitarios, tengan una formación integral en todos los sentidos, en su aspecto personal y profesional. Tanto los profesores como los formadores de profesores deben ser conocedores de la gran influencia que tiene saber aplicar bien los contenidos teóricos y el significado que tiene un buen saber aprendido para el futuro. (Ortiz, 2017, pp. 23-24)

Es necesario que el docente forme en actitudes y valores cívicos al alumnado, para que sean capaces de participar activamente a la comunidad a la que pertenecen. Se deben potenciar estrategias de trabajo para ser capaces de acceder a nuevos conocimientos y capacidades, de adquirirlos, procesarlos y asimilarlos, reflexionando críticamente sobre los fines y el objetivo del aprendizaje. De los profesionales de la educación, se espera que sean personas autónomas y disciplinadas, pero también que sean capaces de trabajar en equipo, participar en grupos diversos y heterogéneos y de compartir lo que hayan aprendido.

Bolívar (2007, p. 27) afirma que "la ciudadanía ha de construirse localmente, ampliando espacios de participación y lugares donde trabajar conjuntamente". La educación cívica no puede consistir sólo en contenidos para aprender en una materia, es decir, en un aprendizaje conceptual, sino en un conjunto de prácticas pedagógicas y educativas que comprenden, al menos, tres componentes: conocimientos, habilidades y actitudes y valores. Como tales, exigen procesos de vivencia en el centro escolar y en la comunidad, que además precisan un cierto grado de consistencia entre ellos. 
La formación para la convivencia ciudadana aparece entonces como una responsabilidad compartida y derivada. Compartida porque las personas somos objeto de formación. Pero, además, la formación para la convivencia ciudadana es una responsabilidad derivada de la sociedad, como factor de desarrollo educativo, y de la educación como factor de desarrollo social, que debe cumplir las exigencias del carácter axiológico de la educación, en lo que corresponde a la formación para la convivencia pacífica, en un marco legal territorializado de relación con el otro (Touriñán, 2009).

Toda actitud positiva debe basarse en la motivación y la confianza, para iniciar y culminar con éxito el aprendizaje a lo largo de la vida. Aplicar lo aprendido y lo vivido, interesarse por aumentar el aprendizaje, y buscar nuevas oportunidades para aprender y ponerlo en práctica, en diversos contextos, es esencial en la formación del estudiantado. La tarea del profesor deberá consistir en ir ampliando su ámbito de explicación y su capacidad de reflexión. Hay que partir de la realidad que conoce el sujeto. La formación que hay que proporcionar en las escuelas tiene que constituir un entrenamiento sistemático en el pensamiento crítico.

\section{Referencias}

Alfaro, Alicia y Badilla, Maynor. (2013). La conceptualización de la Educación Cívica en Costa Rica: aportes profesionales vinculados con esta disciplina. Revista Electrónica Perspectivas, (6), 44-66.

Althof, Wolfgang and Berkowitz, Marvin. (2006). Moral education and character education: their relationship and roles in civic education. Journal of Moral Education, 35(4), 495518.

Annete, John and Mayo, Marjorie. (2010). ¿Taking part? Active learning for active citizenship and beyond. United Kingdom: Niace.

Arbués, Elena. (2014). Civic Education in Europe: Pedagogic challenge versus social reality. Sociology Mind, 4(3), 226-232.

Aspin, David. (2000). Clarification of key terms used in the discussion of morality and the use of the values. In Mal Leicester, Celia Modgil and Sohan, Modgil (Eds.), Moral education and pluralism (pp. 16-31). London and New York: Falmer Press.

Banks, James Albert. (2008). Diversity, Group Identity, and Citizenship Education in a Global Age. Educational Researcher, 37(3), 129-139.

Bialik, Maya, Bogan, Michael, Fade, Charles and Horvathova, Michaela. (2015). Character Education for the 21st century: What should student learn? Boston, Massachusetts: Center for Curriculum Redesign. 
Bolívar, Antonio. (2007). Educación para la ciudadanía algo más que un tema. Barcelona: Graó.

Boyd, Dwight and Arnold, Mary Louise. (2000). Teachers Beliefs, Antiracism and Moral Education: problems of intersection. Journal of Moral Education, 29(1), 23-45.

Boyle, Nigel. (2014). Integratin Global and Local Civic Learning (Early and often). In Jill Reich (Ed.), Civic Engagement, Civic Development, and Higher Education (pp. 73-76). Washington, DC: Briging Theory to Practice.

Browman, Nicholas. (2011). Promoting participation in a diverse Democracy: A Meta-Analysis of College diversity experiences and Civic Engagement. Review of Educational Research, 81(1), 29-68.

Camps, Victoria y Giner, Salvador. (1998). Manual de ciudadanía. Barcelona: Ariel.

Caputo, Dante. (2004). La Democracia en América Latina. Hacia una democracia de ciudadanos y ciudadanas. Buenos Aires: Programa de las Naciones Unidas para el Desarrollo.

Casares, Pilar. (2000). Valores y currículo. In Enrique Gervilla y Andrés Soriano (Coord.), Educación hoy. Concepto, preguntas y valores (pp. 85-106). Granada: Grupo editorial universitario.

Castles, Stephen and Davidson, Alastair. (2000). Citizenship and Migration: Globalisation and the Politics of Belonging. United Kingdom: MacMillan.

Cortina, Adela. (1997). Los Ciudadanos del mundo. Madrid: Alianza.

Crick, Bernard. (1998). Education for Citizenship and the teaching of Democracy in Schools: Final report of the advisory group of citizenship and the teaching of Democracy in schools. United Kingdom: Cualifications and curriculum authority.

Crick, Bernard. (2002). Democracy. Oxford: Oxford University Press.

De Castro, Rosane Michelli y Da Silva, Vandeí Pinto. (2014). Teachers' Formation in Higher Education: Some Aspects under the Light of Education-Work Relations. Creative Education, 5(18), 1684-1689. Recuperado de http://dx.doi.org/10.4236/ce.2014.518187

Delval, Juan. (2012). Ciudadanía y escuela. El aprendizaje en la participación. En Nicolás De Alba, Francisco García Pérez y Antoni Santisteban (Eds.), Educar para la participación ciudadana en la enseñanza de las ciencias sociales (pp. 37-46; Vol. I). Sevilla: Díada.

Díaz, Elías. (2000). Ética pública y estado de derecho. Madrid: Fundación Juan March.

España. (3 de octubre de 1990). Ley Orgánica de Ordenación General del Sistema Educativo. Boletín Oficial del Estado, № 238, pp. 28927 a 28942. 
Garnier, Leonardo. (2013). Proyecto de ética, estética y ciudadanía. Programas de estudios. Estudios sociales y educación cívica. San José, Costa Rica: Ministerio de Educación Pública.

Gelpi, Ettore. (1992). Los Valores y la educación: una perspectiva de la UNESCO. Madrid: MEC.

Gómez Rodríguez, Antonio Ernesto. (2005). Tendencias en la educación cívica en el Siglo XXI. Íber, 44, 7-15.

Gutiérrez, Martha Cecilia y Arana, Diana Marcela. (2012). La formación inicial de los docentes en la educación para la participación. In Nicolás De Alba, Francisco García Pérez y Antoni Santisteban (Eds.), Educar para la participación ciudadana en la enseñanza de las ciencias sociales (pp. 437-442; Vol. II). Sevilla: Díada.

Gutmann, Amy. (1999). Democratic citizenship. In Martha Nussbaum and Joshua Cohen (Eds.). The limits of the patriotism: identity, belonging and world citizenship (pp. 83-90). New york: Reviews.

Gutmann, Amy. (2001). The Democratic education. A political theory of the education. New York: Reviews.

Halstead, Mark y McLaughlin, Terence. (1999). Education in Morality. London and New York: Routledge.

Heather, Derek. (1990). Citizenship: the civic ideal in world history, politics and education. United Kingdom: Longman. Recuperado de https://books.google.co.cr/books?id=2LMhLE40260C\&printsec=frontcover\&hl=es\&sourc $\underline{e}=$ gbs ge summary $r \& c a d=0 \# v=0 n e p a g e \& q \& f=f a l s e$

Hirmas, Carolina y Eroles, Daniela. (2008). Convivencia democrática, inclusión y cultura de paz. Lecciones desde la práctica educativa innovadora en América Latina. Chile: Oficina Regional de Educación de la UNESCO para América Latina y el Caribe.

Ichilov, Orit. (1998). Citizenship and Citizenship Education in a Changing World. United Kingdom. Portland: Or. The Woburn Press.

Jones, Tiffany Mary. (2009). Framing the framework: Discourses in Australia's national values education policy. Educational Research for Policy and Practice, 8(1), 35-57.

Kymlicka, Will. (1995). Multicultural Citizenship: A Liberal Theory of Minority Rights. Oxford: Clarendon Press.

Kymlicka, Will. (2001). Educación para la ciudadanía. En Francisco Colom (Ed.), El espejo, el mosaico y el crisol. Modelos políticos para el multiculturalismo (pp. 251-282). Barcelona: Anthropos.

Lapsley, Daniel and Woodbury, Ryan. (2016). Moral-Character Development for Teacher Education. Action in Teacher Education. 38(3), 194-206. Recuperado de http://dx.doi.org/10.1080/01626620.2016.1194785 
Lickona, Thomas. (1991). Educating for character: How our schools can teach respect and responsibility. New York: Bantam.

Lockwood, Nancy. (2003). Work/life Balance: Challenges and Solutions. HR Magazine, 48(6), 2-10.

Lorenzo, Manuel. (2000). La investigación en la gestión de los centros y el liderazgo. En Manuel Lorenzo y José Antonio Ortega (Coords.), Las organizaciones educativas en la sociedad neoliberal. Registro del sexto Congreso Interuniversitario y de las $V$ Jornadas andaluzas de organización de instituciones educativas (pp. 185-219). Granada: Grupo editorial universitario.

Marchesi, Álvaro. (2000). Controversias en la educación española. Barcelona: Eiunsa.

Martínez Martín, Miquel. (2011). Educación, valores y democracia. Revista de educación, (Extraordinario), 15-19.

Naval, Concepción. (2003). Orígenes recientes y temas clave de la educación para la ciudadanía democrática actual. Revista de educación, (Extraordinario), 169-189.

Naval, Concepción, Print, Murray and Veldhuis, Ruud. (2002). Education for Democratic Citizenship in the New Europe: Context and Reform. European Journal of Education, 37(2), 107-128.

Norris, Pippa. (2002). Democratic Phoenix: reinventing political activism. Cambridge: Cambridge University Press.

Nusbaum, Marta. (2002). Patriotism and Cosmopolitanism. In Joshua Cohen (Ed.), For love of country (pp. 2-17). Boston: Beacon.

Ong, Aihwa. (1999). Flexible Citizenship: The Cultural Logics of Transnationality. London: Duke University Press, Durham.

Ortiz, Eva. (2017). La adquisición de valores para la formación de los docentes en Educación Primaria. Revista Dilemas Contemporáneos: Educación, política y valores, 4(3), 1-28.

Paredes, Eulalia y Ribera, Dolores. (2006). Educación en valores. Barcelona: Tibidabo.

Print, Murray and Coleman, David. (2003). Towards Understanding of Social Capital and Citizenship Education. Cambridge Journal of Education, 33(1), 123-149.

Putnam, Robert. (2000). Bowling alone: the collapse and revival of American community. New York: Simon and Schuster.

Rosaldo, Renato. (1999). Cultural citizenship, inequality, multiculturalism. In Rodolfo Torres, Louis Miron y Jonathan Inda (Eds.), Race, identity and citizenship: A Reader (pp. 253261). Massachusetts: Wiley-Blackwell. Recuperado de http://www.tij.uia.mx/elbordo/vol03/bordo3 ciudadania1.html 
Rychen, Dominique Simone and Salganik, Laura Hersh. (2003). Key Competencies for a Successful Life and a Well-Functioning Society. Cambridge: Hogrefe \& Huber Publishers.

Schmelkes, Sylvia. (1997). La escuela y la formación valoral autónoma. México: Graphos y Entornos.

Schulz, Wolfram. (2012). Educación para la ciudadanía y participación ciudadana. Una presentación del estudio ICCS 2009 y sus resultados. En Nicolás De Alba, Francisco, García Pérez y Antoni Santisteban (Eds.), Educar para la participación ciudadana en la enseñanza de las ciencias sociales (pp. 47-62; Vol. I). Sevilla: Díada.

Ten Dam, Geert and Volman, Monique. (2007). Educating for adulthood or for citizenship: social competence as an educational goal. European Journal of Education, 42(2), 281 298.

Torres, Arturo, Álvarez, Nivia y Del Roble, María. (2013). La educación para una ciudadanía democrática en las instituciones educativas: su abordaje psicopedagógico. Revista Electrónica Educare, 17(3), 151-172.

Thomas, Liz. (2002). Student retention in Higher education: the role of institutional habitus. Journal of Educational Policy, 17(4), 423-442.

Thorberg, Robert and Oguz, Ebru. (2013). Teachers's views on values education: A qualitative study in Sweden and Turkey. International Journal of Educational Research, $59,49-56$.

Touriñán, José Manuel. (2009). El desarrollo cívico como objetivo. Una propuesta pedagógica. Teoría de la educación 21(1), 129-159.

Touriñán, José Manuel. (2010). Familia, escuela y sociedad civil. Agentes de educación intercultural. Revista de Investigación en Educación, (7), 7-36.

Unesco. (1993). Informe mundial sobre la educación. París, Francia: Organización de las Naciones Unidas para la Educación, la Ciencia y la Cultura.

Vargas, Jorge, Rosero, Luis y Seligson, Mitchel. (2006). Cultura política de la democracia en Costa Rica. Costa Rica: Centro Centroamericano de población de la Universidad de Costa Rica.

Wringe, Colin. (2006). Moral Education. Beyond the Teaching of Right and Wrong. Dordrecht, The Netherlands: Keele University, U.K.

Wu, Xianfeng and Oldfield, Philip. (2015). How "Civic" the Trend Developed in the Histories of the Universities. Open Journal of Social Sciences, 3(6), 11-14. Recuperado de http://dx.doi.org/10.4236/jss.2015.3600

Zvokey, Daniel. (1990). Objetivity and moral judgment: Towards Agreement on a moral education Theory. Journal of moral education, 19(1), 14-23. 